OPEN ACCESS

Edited by:

Martin Bojowald,

Pennsylvania State University,

United States

Reviewed by:

Jorge Páramos,

University of Porto, Portugal

Vyacheslav Ivanovich Dokuchaev, Institute for Nuclear Research (RAS),

Russia

${ }^{*}$ Correspondence:

Ricardo B. Ferreira

rbferreira@isa.ulisboa.pt

Specialty section:

This article was submitted to

Cosmology,

a section of the journal

Frontiers in Astronomy and Space

Sciences

Received: 09 June 2017 Accepted: 14 September 2017

Published: 27 September 2017

Citation:

Ferreira RB and Ferreira JB (2017) The

Live Universe. A Biologist's

Perspective.

Front. Astron. Space Sci. 4:17.

doi: 10.3389/fspas.2017.00017

\section{The Live Universe. A Biologist's Perspective}

\author{
Ricardo B. Ferreira ${ }^{1 *}$ and João B. Ferreira ${ }^{2}$ \\ ${ }^{1}$ Disease and Stress Biology, Instituto Superior de Agronomia, LEAF, Universidade de Lisboa, Lisbon, Portugal, ${ }^{2}$ Hospital de \\ Santa Maria, Centro Hospitalar Lisboa Norte, Lisbon, Portugal
}

Astrobiology looks at all aspects related to life in places other than the Earth, including its biomolecular building blocks and suitable environmental conditions. In the present article, a different approach is followed: a comparative analysis between Astronomy and Biology as discrete domains of science. Remarkable similarities exist between these two apparently widely separated and multidisciplinary fields. Both are driven, from beginning to end, by thermodynamics. Their evolution is studied to a very reasonable degree of accuracy, from beginning to the present day, by analyzing data which were "frozen" in the past. Yet we cannot predict where and how they will go from here. A major difference is that in Biology, unlike Astronomy, we can see and analyse the present (or, more accurately, the immediate past). While the Big Bang is widely accepted as the origin of our universe, the debate about its ultimate fate is far from settled. A plethora of cosmological models has been proposed, many involving the concept of a multiuniverse. The observation that the rate of expansion of the universe is apparently accelerating further boosts the discussion. Entropy may act as a driving force behind the increasing rate of expansion, with nothingness as the maximum possible entropy our universe gets. Using biological systems as an analogy and adopting a broad definition for life, we may speculate the existence of a living multiuniverse, capable of natural evolution, in which each individual universe spontaneously goes through birth, development, reproduction, aging and death. The possible roles of supermassive black holes (SMBHs) and human-like intelligence on the future evolution of our universe are briefly discussed.

Keywords: astronomy, biology, evolution, life, multiuniverse, black hole

\section{INTRODUCTION}

Astrobiology looks at all aspects related to life in places other than the Earth, including its biomolecular building blocks and suitable environmental conditions. Astronomy and Biology, on the other hand, are generally regarded as widely separate fields of science. Shortening or even bridging the wide gap between them may not be an easy task due to their specific multidisciplinary nature, their widely different approaches, and the sheer amount of knowledge gathered in each field. As a result, many astronomical issues are somewhat inaccessible to the biological community and vice-versa, despite the existence of several potentially overlapping areas of reasoning between them. The present article aims at tentatively filling this gap. This may compromise to some extent the detail of the analysis presented in this article, something which may be counterbalanced by surprising and eventually rewarding observations. 
While the Big Bang theory for the origin of our universe has been widely accepted, the debate about its ultimate fate is far from settled. As a result, a plethora of cosmological models has been proposed, such as the "Oscillating universe" and the "Evolving universe," with several of them involving the concept of a multiuniverse (Battersby, 2006). The observation that the rate of expansion of the universe is apparently speeding up, as well as data obtained recently from the cosmic microwave background $(\mathrm{CMB})$ radiation, support the view that our universe will most likely expand forever (Perlmutter et al., 1999; Riess et al., 1999, 2016; Scrimgeour et al., 2012; Davis, 2014). A considerable number of factors, such as entropy, gravity and supermassive black holes (SMBHs), will certainly play a leading role in determining the future evolution of the universe.

As specified by the 2nd Law of Thermodynamics, the role played by entropy in nature cannot be overstated. Proteins, for example, the remarkable polymers which lie behind virtually all events which take place in biological systems, have evolved to attain conformations that extraordinarily and simultaneously match those (i) which exhibit biological activity and (ii) which are more stable under biological conditions. Such conformations are essentially achieved by the entropy-driven, hydrophobic collapse. Nothing less than this would be expected for the involvement of entropy in the universe/multiuniverse.

The observation that our universe seems to fulfill the premises behind the concept of "life as we know it" may allow moving one step beyond the "Evolving universe" and propose the notion of a "Living universe." The hypothesized life-style evolution of our universe most probably raises more questions than it settles. The main purposes of this article are therefore to stimulate discussion and bring selected concepts from other areas of science, in an attempt to shed light in some of the most debated issues of modern cosmology. In addition, a certain degree of biologically-oriented speculation was launched with the objective of promoting and introducing new ideas to encourage debate and "brain storming."

\section{NATURAL EVOLUTION IN ASTRONOMY AND BIOLOGY. FROZEN IMAGES FROM THE PAST}

Our knowledge about natural evolution in Biology relies largely on fossil data. Thus, when we look at a fossil record, we see a wide range of organisms "frozen in time," from different periods and which provide valuable information with environmental and biological significance. Therefore, fossil records are like pieces of a puzzle that explain what we see today and from which we extract useful information from the past. Although we cannot predict how life will be in the future (at least in a broad, meaningful sense), we can easily "look" both at the present and the past.

Current evidence shows that the universe is expanding at an accelerated rate, so that galaxies in general recede from us in all directions: nearby objects recede slowly whereas more distant ones recede rapidly. Therefore, when an observer looks through a powerful telescope to a very distant galaxy, the light received by the telescope shows that distant galaxy when it was closer to the observer, at a time when the universe was younger and smaller. The apparent size of this galaxy will be much bigger than what would have been predicted if the observer could look at it as it is right now. In contrast, distant galaxies are much dimmer than expected due to the very long time light took to reach the observer-it should be borne in mind that space is expanding right across the path light travels in our direction from the emitting source, meaning that many photons' paths become stretched and spread out over a wide area, whereas some photons are invariably lost. The most distant galaxies visible with the Hubble Space Telescope were only a few billion light-years away from us when they emitted the photons we are now receiving. Therefore, they look much larger than expected, as if they were now about 2 or 3 billion light-years away, but are as faint as if they were about 350 billion light-years away, even though they are much closer.

In similarity to Biology, when we use a telescope to observe both nearby and distant galaxies we are also looking back in time: we see each galaxy as it was when the corresponding light was emitted. However, unlike in Biology, it is not possible to determine in what way that galaxy has evolved to the present day, since it may now be farther away or closer to us, or even not existent anymore. As in fossil records, we receive the image of each galaxy as "frozen in time," with the "geological period" varying immensely among galaxies. The closest galaxies are seen as they were in a recent past (e.g., we see Andromeda galaxy, NGC 224 , as it was about 2.5 million years ago), while the farthest ones reveal themselves as they were in a distant past (e.g., GN-z11, the most distant galaxy known, recently shown by the Hubble Space Telescope to be 13.4 billion light-years away; Wu et al., 2016). In connection to this, many deep field photographs taken by the Hubble Space Telescope show hundreds of galaxies as they were at vastly different times in the past. It is then possible to estimate the recession velocity between any two galaxies while being observed at distinct eras of the universe, in much the same way as we may predict the evolution which took place between two fossilized organisms which lived in distinct eras of the past.

As in Biology, it is not possible to predict how the universe will evolve from now unless we assume conditions will remain the same as in the past. However, in marked contrast to Biology (and with the exception of the solar system and nearby stars), it is not possible to see, determine or foresee how the universe is at present, even within the Milky Way. This implies that any galaxy we observe may have evolved into a "complete new species."

The biological correspondence to looking at a Hubble Space Telescope picture comprising several, deep space galaxies is, from our point of view, a tablet of fossils including a primitive cell $\mathrm{c}$. 3.7 billion years old (i.e., the oldest solid fossil evidence for life although molecular genetics suggests the first life forms may have existed as early as $4.2-4.3$ billion years ago), a 2.1 billion years old primitive eukaryote, and a 1.7 billion years old multicellular organism.

Knowledge about universal evolution is, as with biological evolution, inevitably built on data gathered at widely different times in the past. On the other hand, we have no way to accurately predict how evolution will proceed in the future. 
There is, however, a striking difference between Astronomy and Biology: in biological evolution we can look, analyse and study the present (or, strictly speaking, the immediate past), whereas the astronomical present is out of reach.

\section{LITTLE HOPE FOR AN OSCILLATING UNIVERSE}

Friedmann (1922) was the first to propose a closed cyclic universe. Many other cyclic models have been suggested ever since. They are philosophically appealing, as noted by Steven Weinberg (1977) "the oscillating model [...] nicely avoids the problem of Genesis." Indeed, they address (but do not solve completely) a major challenge left unsettled by the big bang inflationary model, i.e., "What happened before the Big Bang?"

Khoury et al. (2001) proposed the ekpyrotic universe, a cyclic universe model suggesting a complete cosmological history including both past and future, in which our universe is caught in an eternal cycle of fiery birth, cooling and rebirth. Though no physical experiment has been performed to demonstrate how it could ever happen, some scientists speculate that a Big Crunch would not necessarily signal the end of everything. The cosmological model combining both the Big Bang and the Big Crunch as part of a cyclic process, i.e., the idea that Bangs follow Crunches in a never-ending cycle, became known as the "Oscillating universe," which posits that the universe would simply expand and contract (or bounce) forever, and that the universe we live in would exist between a Big Bang and a Big Crunch.

In the oscillating universe hypothesis the Big Bang is regarded as a phase transition from a metastable state into another more stable state accompanied with release of energy (Chensky, 2013): some aspects of the former universe are irretrievably lost through the Big Bang, and no two universes in the sequence would ever be the same owing to "cosmic forgetfulness" (Bojowald, 2007). In other words, our present universe may have arisen from the final stage of collapse of an all-in black hole containing the remnants of a prior universe by bubbling out of that black hole's core into a Big Bang. As the new universe gradually expanded from a very high density and high temperature state, matter slowly coalesced into stars, solar systems, galaxies and new black holes, changing from a very high prevalence of light elements to a gradual build up of heavier elements. Black holes would in turn start to grow and coalesce to form bigger black holes, culminating in a massive merger, and thus beginning a new cycle.

Conventional theories assume that the Big Bang was preceded by a singularity, where the laws of physics break down. From a pure and unchanging thermodynamic point of view, phase transitions in an oscillating universe should be accompanied by energy release (e.g., entropy increase), as initially stated by Tolman (1934). In this sense, old and new universes cannot be identical. In order to circumvent the constraints imposed by classical thermodynamics, Tolman (1934) changed the view from a succession of identical cycles to a periodic universe in which each new cycle became greater than the previous cycle, both with respect to the period and the maximum value of the curvature radius, albeit with a gradual but continuous increment in entropy from one cycle to the next (Tolman, 1934). This increase in entropy from cycle to cycle allows for only a limited number of cycles. Therefore, in the oscillating model, some information seems to be lost even if the laws of physics would hold during phase transition, thus creating an "information horizon" which stops us from fully knowing all the previous universe's properties. As stated by Bojowald (2007), "An eternal recurrence of the same is prevented by intrinsic cosmic forgetfulness" or "A memory of certain aspects of the universe before the Big Bang is lost while transiting through the Big Bang." It should be taken in consideration that cosmological models are typically based on a number of assumptions, are constrained by theoretical limitations and are not fully corroborated by experimental evidence. Therefore, our limitations concerning the oscillating model derive from the observation that "complete predictions and explanations of observations can only be made for the finite part starting after the Big Bang," since the singularity is a theoretical limitation, rather than a physical beginning (Bojowald, 2007). For this reason, it is not possible to ensure that old and new versions of universes at each transition on a cyclic model are not identical.

The oscillating universe models take into account cosmological horizon and flatness issues, without requiring superluminal expansion (inflation) or multiuniverse. However, as appealing as it may be, the oscillating universe theory leaves unanswered a fundamental question: where did the original universe come from? This issue has yet to be solved. Alternatively, the oscillating universe may exist since ever and forever.

Many serious drawbacks of both observational and theoretical nature have been claimed against oscillating universe models (Kragh, 2013). Nevertheless, these models continue to call the attention of several cosmologists, who devise alternatives or adopt recent findings to overcome the raised obstacles.

\section{THE EVOLVING UNIVERSE REVISITED: A RENEWED VERSION OF THE EVOLVING UNIVERSE}

In 1997, Lee Smolin added a twist to the story of the universe by proposing cosmological natural selection: in a generation-togeneration way, a population of universes (including the one we live in) are tuned toward the production of black holes. This idea, known as the "Evolving universe" model, was suggested at a time when many physicists believed the rate of expansion of the universe was decreasing and would eventually come to a halt, before collapsing into a Big Crunch (section "Little Hope for an Oscillating Universe", above).

The "Evolving universe" model fits well within the notion of multiuniverse, which lies at the borderline between physics and philosophy. Many cosmologists now include the realms of the multiuniverse within physics rather than metaphysics, but critics of the multiuniverse disagree and consider them as basically non-scientific (Kragh, 2009). The last one or two decades have witnessed an intense debate on the position our universe occupies 
in a supposedly much larger picture, the multiuniverse, as well as on the possibly related concepts of black holes, wormholes and white holes.

Gale (1990) proposed a simple classification of multiuniverse models in three groups: spatial multiuniverses (e.g., the models considered in section "A Live Universe: One Step Beyond the Evolving Universe", below temporal multiuniverses (comprising the cyclic models), and models with universes of other dimensions.

\section{Black Holes}

The existence of black holes was first demonstrated in 1916 by Karl Schwarzschild. The origin and fate of black holes is inexorably dictated by thermodynamics and has been a hot topic for the last century. According to current knowledge, those with initial masses between one hundred and one hundred thousand solar masses are believed to have formed early in the "life" of the universe. With time, these large black holes grew up by sucking matter from the surrounding galactic material and/or by merging with other black holes, until some of them reached masses equivalent to millions or even billions of suns. At this stage, they become known as $\mathrm{SMBH}$. Whatever the scenario, $\mathrm{SMBH}$ are likely to play an important role in determining the ultimate fate of our universe.

The ultimate fate of our universe has long been considered to depend on the balance between the momentum of expansion imparted by the Big Bang and the pull exerted by gravity. The accelerated expansion rate that our universe seems to go through at present may cause the universe to spread out ceaselessly. The Big Rip cosmological model, initially formulated in 2003 by Caldwell and colleagues and recently supported by novel mathematical formulation (Disconzi et al., 2015), proposes that the universe will expand without limit, with dark energy eventually becoming so unbearably strong that the matter of the universe, from stars and galaxies to atoms and subatomic particles, will be progressively torn apart at a certain time in the future (Caldwell et al., 2003). Other cosmological models have been proposed in which the universe will also expand forever. In the Big Freeze scenario, gravity slows down the expansion but does not succeed in stopping it. As the "fuel" in all-star generations becomes exhausted, the universe gradually grows cold and dark as it expands, leaving behind just black holes which, eventually, will evaporate as too space gets ever colder.

However, assuming that a Big Crunch will occur sometime in the future, a distinct SMBH-dependent sequence of events should take place: SMBHs present at galaxy centers could eventually "swallow" the entire galactic mass of gas, dust and stars, and then float through space until they encounter and merge with other giant holes that have already devoured their own former galaxies. Relentless gravity will subsequently move them ever closer one another and join together many galactic black holes into a colossal black hole.

SMBHs with masses of around 10 billion suns have been found at the center of large galaxies, where they grow larger as they feast on surrounding matter. They are believed to be relatively common in the universe (Venemans, 2015). At least some were already present 13 billion years ago. Among the largest SMBHs found to date is one with a mass of 12 billion suns from a time when the Universe was less than 900 million years old ( $\mathrm{Wu}$ et al., 2015), and one other was found with a mass of 17 billion suns at the center of the large NGC 1600 galaxy located in a sparsely populated area of the universe 200 million light-years away (Thomas et al., 2016). News published in 11 February 2016 at the Hubble Space Telescope site (https://www.spacetelescope. org/news/heic1602/) indicates that the record holder is, so far, a 21 billion suns-SMBH, which is located in the giant elliptical galaxy NGC 4889, about 300 million light-years from the Earth. For comparative purposes, the Milky Way harbors a central SMBH equivalent to 4-5 billion solar masses (Venemans, 2015).

Black holes are believed to contain a singularity (i.e., a point occupying no space but infinitely dense and infinitely hot, thus creating a bottomless pit in the fabric of space-time), the nature of which is not completely understood. Current physical theories are considered to break down near singularities (Begelman, 2003; Kim and Park, 2015). Under such conditions, the structure of ordinary matter is disrupted. Assuming SMBHs are permanently "eating" the universe from within, growing almost unlimitedly, and combining that with a spatially flat, expansioning universe, the following scenario may be hypothesized: SMBHs undergo a dramatic change once they reach a critical mass/pressure stage: they originate a big bang for a newly born universe by connecting such singularity to a distinct set of dimensions, operating as a linkage between "father and son" universes. As a result, the unstructured matter elements escape (under a tremendous amount of pressure) into another "space" at zero or low pressure. Matter and energy are eventually projected in all directions at great but varying speeds (eventually ensuring that the offspring universe will comply with the Hubble law; Hubble, 1929) as pressure drops dramatically: a big bang takes place and a new universe is born.

Soon after big bang, the unstructured matter reassembles into light atoms (i.e., ordinary hydrogen) in the newly born universe. As the universe ages and stellar fuel is consumed, successively heavier atoms are gradually accumulated inside stars, thus powering the evolution of the universe, a part of which we may suppose to be life. Over very long periods, the increasing production of heavier atoms will contribute to matter gradually clumping and collapsing once again inside black holes, under the tearing effect of gravity.

Thus, by ripping apart atomic structures, $\mathrm{SMBHs}$ could eventually "give birth" to juvenile universes with a renewed atomic composition. This might just be the "evolving" counterpart of Bojowald's cosmic forgetfulness.

This speculative hypothesis meets an immediate limitation: unless matter and/or energy are created/introduced somewhere/somehow along this process, their total amount in each universe would inevitably decrease continuously at every progenitor to offspring transition.

\section{White Holes}

The problem of matter/energy discontinuity was addressed by Igor Novikov, who showed that general relativity has space for white holes (Novikov, 1964). White holes, whose existence has yet to be demonstrated, correspond to hypothetical regions of 
space-time which cannot be entered from the outside. As the reverse of black holes, white holes permanently blow out streams of matter and light. Attempts are now underway to find them at the center of galaxies.

Poplawski (2010) suggested that the reverse of the formation of a black hole by the gravitational collapse of matter through the event horizon, i.e., the blast of emerging matter from a white hole through the event horizon, is also possible.

\section{Wormholes}

In 1957, John Wheeler introduced the term and concept of wormhole (Regge and Wheeler, 1957). For example, traversible wormholes were first studied by Morris and Thorne (1988), and then became accepted as a scientific topic (Visser, 1995). Subsequently, Hayward (1999) proposed a unified theory of black holes and traversible wormholes, arguing that the two are dynamically interconvertible, and that traversible wormholes are understandable as black holes under negative energy density.

\section{Black Holes, Wormholes, and White Holes}

The proposed evolving universe model ties hands with the concepts of black hole, white hole and wormhole. Large, spinning black holes have already been reported as possible portals/passageways to other universes (Hawking et al., 2016). It has also been suggested that black holes could be positioned at the end of a wormhole connecting two separate regions of the universe, or even two different universes, sucking matter from one end (i.e., behaving as a black hole) and injecting it in the other (i.e., behaving as a white hole). In this way, white wholes could be responsible for occasional injections of matter in our universe or, going one step beyond, the big bang which created our universe could have been the result of a white whole. Indeed, a 2012 article suggested that the big bang itself is a white hole, with all the matter being ejected as a single pulse, or "Small Bang." The same authors suggested white holes as the best explanation for a new group of $\gamma$-ray bursts that appear in voids, which are relatively close to Earth, but surprisingly lack any supernova emission (Retter and Heller, 2012). Nevertheless, theoretical evidence so far suggests that anything falling into a rotating black hole would block the wormhole.

Albert Einstein predicted that space-time singularities, where the classical concepts of space and time breakdown (Kim and Park, 2015), occur at the center of black holes. Wormholes arrived as viable alternatives to singularities. Shinkai and Hayward (2002) studied the stability of a traversible wormhole and concluded that the wormhole throat suffers a bifurcation of horizons and either explodes to form an inflationary universe or collapses to a black hole, if the total input energy is respectively negative or positive. Haggard and Rovelli (2015) have recently shown that there is a classical metric where matter collapses into a black hole and then emerges from a white hole, suggesting that a black hole can quantum tunnel into a white hole.

The existence of wormholes in space-time has been proposed, but they require small amounts of exotic matter for stability and to prevent them from collapsing (Morris and Thorne, 1988) or for wormhole maintenance and sustainability (Visser, 1995; Fewster and Roman, 2005). Exotic matter, which remains a mystery, is mathematically defined as matter which violates our perception of energy. Therefore, if an observer detects a region of negative energy density, that should correspond to exotic matter (Azreg-Aïnou, 2015). Alternatively, Lobo and Oliveira (2009) constructed traversable wormhole geometries in the context of $f(R)$ modified theories of gravity. These theories, first proposed in 1970 by Hans Buchdahl, constitute a type of modified gravity theory which generalizes Einstein's general relativity (Buchdahl, 1970). By introducing arbitrary functions, a wide range of phenomena can be explained with no need to introduce unknown forms of matter or energy. Many are ruled out simply as a result of observational data collected or due to theoretical constraints.

The black hole information paradox, a "black holy brother" of cosmic forgetfulness, the notion that information cannot be destroyed, and the concurring belief that the laws of physics breakdown at singularities could at least in part be solved by black-to-white hole conversions (Hawking, 1975; Hawking et al., 2016).

The big bang theory assumes the universe started as a singularity, but no plausible evidence has explained how such a singularity formed in the first place. Such singularities have been suggested to be viable alternatives to wormhole linkages to other universes (Poplawski, 2010).

Easson and Brandenberger hypothesized that the known universe may have been born out of a black hole (Easson and Brandenberger, 2001). Poplawski (2010) went a step further and suggested that our universe may be located inside a wormhole which is itself a part of a black hole located in a larger universe. In an article entitled "Out of the White Hole: A Holographic Origin for the Big Bang," Pourhasan and colleagues proposed in 2014 another theory, in which a four-dimensional star from another universe collapsed,(Pourhasan et al., 2014) forming a 4-D black hole, which in turn could have provided a passage, originating our universe (Hawking et al., 2016).

Poplawski (2010) further proposes that matter may be escaping other universes through SMBHs and discharged into our universe by white holes.

\section{Appealing Aspects of the Renewed Version of the Evolving Universe}

The renewed evolving universe cosmological model has four appealing aspects:

(1) It explains the apparently exquisite fine-tuning of the physical constants and cosmological parameters governing our universe.

As noted by Barrow (2008), "the coincidences encountered by physicists in the cosmos are perplexing, not to say astonishing." One of the basic features of ordinary matter in our universe are the values assumed by a set of fundamental physical constants and cosmological parameters, which seem to have been finetuned, i.e., each of them must lie within a very narrow range to allow matter to coalesce under the effect of gravity up to ignition in star cores, thus allowing energy generation and the formation of chemical elements beyond lithium in the periodic table, such as carbon (Davies, 1982; Press et al., 1983). If any of these fundamental constants were only slightly different, the 
universe would be unlikely to contain astronomical structures, elemental diversity, or life as we know it (Carr and Elli, 2008).

One other example with much relevance to biology is provided by carbon, an element capable of combining with a very large variety of other elements to form a colossal array of molecules. Carbon is the 4th most abundant element in the universe, after hydrogen, helium and oxygen. About one fifth of this carbon is believed to comprise polycyclic aromatic hydrocarbons (PAHs), a huge family of compounds containing up to hundreds of carbon atoms, which are both widespread and abundant throughout the universe. However, the abundance of carbon in the universe would have been only a tiny fraction of that were it not for a remarkable multiple coincidence in resonance levels (Barrow, 2008), namely the existence of a precisely-tuned excited state of the carbon nucleus known as the Hoyle state. Hoyle state carbon decays into ordinary carbon-12, which is essential to all biochemical processes associated with life. The existence of this excited carbon nucleus was predicted in 1953 by astronomer Fred Hoyle and its existence was subsequently demonstrated experimentally.

It has been assumed that the exquisite values adopted by the physical constants in the present universe arose by mere chance, in a highly unlikely event. Other experts hold that although the physical constants were somehow tuned, they could have adopted other values leading to viable universes, albeit rather different from our own (Carr and Elli, 2008). They reckon that instead of constants undergoing a fine-tuning process, it was our evolution that was fine-tuned by them. Quoting Victor Stenger, "The universe is not fine-tuned for humanity. Humanity is fine-tuned to the universe."

(2) If a number of SMBHs originated newly formed universes and shuffled matter from the parent universes into their offspring, this phenomenon would have gradually loosened the restraint imposed by gravity on the parent universes enlargement rate. The consequent reduction in overall density could in part explain the increased acceleration in the rate of expansion of our universe, thus reducing our dependence on dark energy.

(3) Assuming that matter is gradually escaping from the universe via SMBHs into its offspring universes, an aging trend is expected in the composition of the parent universe. Figure 1 suggests this could be the trend behind our universe's change in composition over time, with an increase in dark energy at the expense of ordinary and dark matter. On its turn, this aging trend might also help explaining the actual accelerating expansion rate of our universe.

(4) The cold spots detected in the most recent $\mathrm{CMB}$ radiation maps (Aron and Grossman, 2013). The Planck spacecraft (ESA), whose detectors are 10 times more sensitive than those of its predecessor, the WMAP (NASA), has created the highestresolution map of the entire $\mathrm{CMB}$ yet, confirming the presence of an enigmatic alignment of the universe, the cosmic "Axis of Evil," and a mysterious cold spot, which has been speculated to constitute a scar or mark made by a collision with another universe (Figure 2A). In 2011, Feeney and colleagues suggested the presence of four candidate "bruises" (Figure 2B). In light of a renewed Evolving universe hypothesis, these scars could

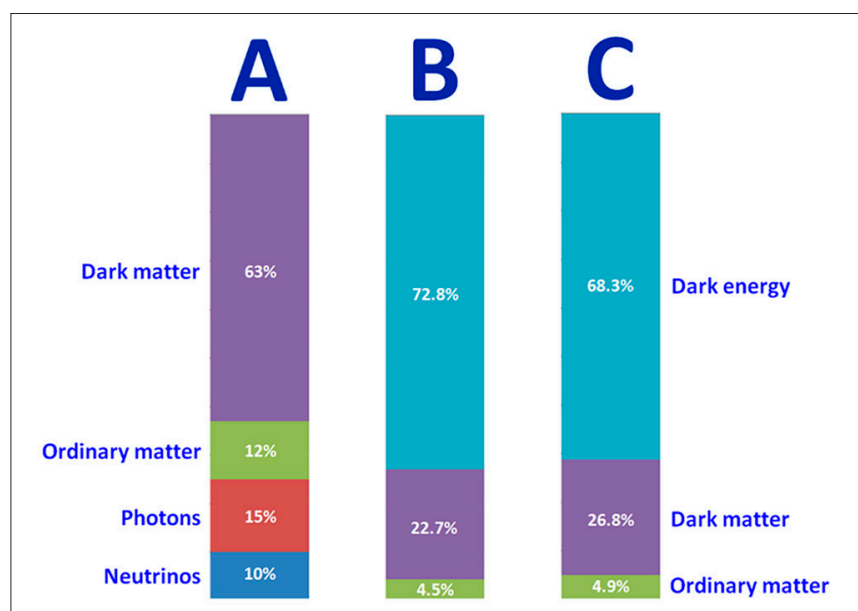

FIGURE 1 | Approximate composition of the universe, (A) when it was 380,000 year-old, and of today's universe, (B) before and (C) after ESA's Planck satellite. Although matter alone may have made up most of the early universe, dark energy must now be added to explain the universe's persistent flatness. Credits: NASA and ESA.

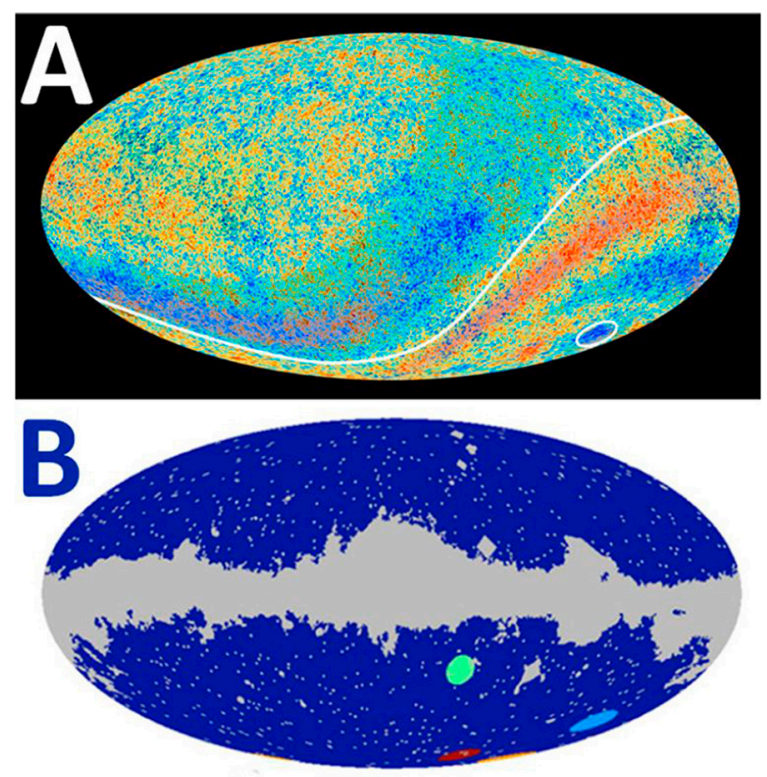

FIGURE 2 | Map of the cosmic microwave background (CMB) radiation. (A) The Axis of Evil and a mystery cold spot stand out in this enhanced version of the best map yet of the CMB radiation obtained by the ESA Planck spacecraft. Data released in 21 March 2013. Credits: ESA-Planck collaboration (B) Four candidate "bruises" are in the lower-right quadrant of this all-sky map of the $\mathrm{CMB}$, in green, light blue, red and orange (bottom edge of image). Data from NASA's Wilkinson Microwave Anisotropy Probe (WMAP) and from Feeney et al. (2011). Credits: The American Physical Society, http://dx.doi.org/10.1103/ PhysRevD.84.043507.

be considered remnant belly buttons, where SMBHs from our universe gave birth to offspring universes.

The cold spot shown in Figure $2 \mathbf{A}$ remains essentially a mystery since it was discovered in 2004 by both the NASA's 
WMAP and the ESA Planck spacecraft. A considerable number of explanations have been suggested, ranging from experimental error or sheer coincidence, to the axis of rotation of the universe (Jaffe et al., 2005), gravitational evolution of a cosmic texture (Cruz et al., 2007), alternative inflationary models (Sánchez, 2014) or a cosmic bubble collision (McEwen et al., 2012). The suggestion that the cold spot could be a hint for the presence of another nearby universe was naturally received with enthusiasm by the scientific community in general.

Meanwhile, an unusually large empty volume of space was tracked between us and the cold spot imprint in the $\mathrm{CMB}$, meaning the imprint of a supervoid via the Integrated Sachs-Wolfe (ISW) effect (Inoue and Silk, 2006). This has been repeatedly reported from 2005 onwards (Marcos-Caballero et al., 2016). In 2015, Szapudi and colleagues provided a possible explanation for the cold spot when they studied the supervoid aligned with it (Szapudi et al., 2015). This huge empty region was named Eridanus supervoid since it is located in the constellation Eridanus (bottom right of Figure 2A). It corresponds to an exceptionally cold and large supervoid in the CMB map (c. 1.8 billion light-years in diameter), located c. 3 billion light-years away from the Earth. It is the largest feature ever identified by man in the mass distribution of the universe, with a galaxy density much lower than elsewhere.

It is considered highly unlikely that the cold spot and the Eridanus supervoid are aligned (when viewed from the Earth) due to sheer coincidence. Au contraire, it has been shown that large voids produce cold spots on the CMB radiation (Cai et al., 2014). These observations suggest a physical connection between both phenomena. However, even if they are linked, various studies have now shown that the presence of the supervoid does not fully explain the cold spot (Nadathur et al., 2014; Marcos-Caballero et al., 2016; Nadathur and Crittenden, 2016). More recently and using more accurate data, Mackenzie et al. (2017) presented evidence which revealed the apparent absence of a supervoid in the direction of the cold spot. Without a supervoid in the way, the same team estimated a probability of ca. 0.02 for the cold spot to have appeared by random chance. The significance of the cold spot as an anomaly will certainly continue to be a hot topic for intense debate.

It is important to emphasize that, as referred above, many cosmologists view the "Axis of Evil" and/or the CMB cold spot simply as statistical artifacts (eventually related to cosmic variance, i.e., the implied bias of having a sample size of only one universe, our own) or local phenomena.

Take a look at the renewed "Evolving universe" under the scope of entropy, and we can foresee a bigger void. If entropy acts as a driving force behind the universe's increasing rate of expansion and considering the possibility of matter escaping from the heart of SMBHs, then one might picture what will become of our universe when it reaches the point of maximum entropy: nothing!

\section{Could an Entropic Factor Lie Behind the Birth of New Universes? \\ Basics on Entropy}

The role played by entropy in life and in the evolution of the universe cannot be overstated. The second law of thermodynamics states that the total entropy of any isolated system tends to increase over time, approaching a maximum value.

To a good approximation (these concepts were initially formulated for isolated systems containing ideal gases), let us use a simple, everyday example to introduce the concepts of entropy and fluctuation. Consider the following isolated system at room temperature: a glass containing an imaginary vertical membrane in the middle, filled with water (e.g., $180 \mathrm{~mL}$, or ca. $6.023 \times 10^{24}$ water molecules), and containing glucose molecules (e.g., $1.8 \mathrm{~g}$, or ca. $6.023 \times 10^{21}$ glucose molecules) exclusively on one side of the membrane (i.e., in $90 \mathrm{~mL}$ of water).

As a result of the second law, the glucose molecules will gradually diffuse, moving continuously, colliding with each other (as well as with water molecules and the walls of the container), following an apparent chaotic and unpredictable pattern which, on average, will lead to ever increasing disordered distributions, until a maximum is reached when the glucose molecules become evenly scattered over the $180 \mathrm{~mL}$ water. There is an astronomical number of highly disordered distributions which correspond to this maximum, meaning that the glucose molecules will continue to move constantly, erratically and in a random way: the microstates will invariably change after the macrostate attains the point of maximum stability. Under these conditions, at each moment in time, each glucose molecule will occupy a distinct position and its momentum will so continually change as it collides with other molecules. The number of molecules is so large that the details of the motion of each individual glucose is basically irrelevant to the behavior of the solution as a whole.

The number of possible microscopic states (microstates) concerns the number of ways in which the glucose molecules can be rearranged, so that the glass of water (i.e., the macrostate) looks exactly the same. Each individual microstate is defined by the positions and momenta of all glucose molecules. Therefore, the higher the number of microstates within a given macrostate, the higher is the entropy of the system. When less microstates are available within the macrostate (e.g., the glucose concentrated in $90 \mathrm{~mL}$ of water), the system has lower entropy. Therefore, there are far more ways for a system to be high entropy than low entropy, i.e., it is overwhelmingly probable for the glucose molecules to spread out, filling the entire volume of water evenly.

The entropy $S$ is defined as:

$$
S=k_{B} \ln W
$$

Where $k_{B}$ is the Boltzmann's constant, which is equal to 1.38065 $\times 10^{-23} \mathrm{~J} \mathrm{~K}^{-1}$, and $W$ is the number of possible microstates corresponding to a given macrostate. Quoting Max Planck, "The logarithmic connection between entropy and probability was first stated by L. Boltzmann in his kinetic theory of gases."

Determining the maximum entropy the universe will get and calculating the difference between the maximum and the actual 
entropy of the universe is especially important, since it would provide a measure of the free energy left in the universe to drive all processes. However, estimating the maximum entropy our universe will get is not an easy task for a number of reasons. First, we are unsure whether the laws of thermodynamics hold at very large scales of our universe. If they do, the overall entropy of the universe can only decrease in a sustainable manner if our universe is not an isolated system (i.e., if energy and/or matter are allowed to exchange with some other structures). Second, to estimate the total entropy of the universe we would have to know the size of it, but our universe seems to be far larger than we can see. Usually, a representative volume of the expanding, visible universe is taken as a supposedly isolated system. A reasonable choice for this comoving volume is the comoving sphere that presently corresponds to the currently observable universe (Egan and Lineweaver, 2010). Alternatively, a certain volume of the expanding visible universe comprising several thousand galaxies and black holes contained within an imaginary sphere is considered. However, none of these approaches considers the entire universe.

\section{Living Organisms and Black Holes Are Open Systems} Living organisms are highly ordered superstructures, characterized by a low level of entropy, with no violation of the second law because they are open systems. To maintain their entropy low, living cells require a continuous supply of energy (e.g., from respiration), most often in the form of ATP. However, when considered within isolated systems containing their environment, the overall entropy increases because living cells "export" entropy into their extracellular milieu. Many examples of this are found in the biological systems, some of which were gracefully illustrated in 1945 by Erwin Schrödinger in his famous book "What is Life? The Physical Aspect of the Living Cell." Two well-known proteomic examples are given below on the entropic nature of both protein folding and protein crystallization.

On a first stage, the universal mechanism of protein synthesis produces unfolded polypeptides, each of which may assume, from a theoretical point of view, an infinite number of possible conformations, as elegantly illustrated by Levinthal's paradox (Levinthal, 1968; Zwanzig et al., 1992). Remarkably, each protein has evolved to fold and assume only a few conformations under cell conditions that, as referred above, match those which simultaneously exhibit biological activity and are thermodynamically more stable.

The whole process of protein folding is directed by the wellknown equation:

$$
\Delta G=\Delta H-T \Delta S
$$

which relates energy with enthalpy and entropy. Transition of a polypeptide from a highly entropic, unfolded state into an ordered globular and compact structure $\left(\Delta S_{\text {protein }}\right)$ is at first sight unfavorable from a thermodynamic point of view. Assuming a low contribution of enthalpy to the overall $\Delta G$ negative value, folding is then driven by a proportionally larger increase in entropy $\left(\Delta S_{\text {solvent }}\right)$ generated by the release of water molecules from the structured clathrate cages that form around the polypeptide hydrophobic amino acid side chains exposed to the surrounding aqueous medium. Therefore, the above equation should be better written as follows when protein folding is considered:

$$
\Delta G=\Delta H-T\left(\Delta S_{\text {protein }}+\Delta S_{\text {solvent }}\right)
$$

$\Delta G$ is invariably negative and most polypeptides fold spontaneously on their own into biologically active proteins.

Exactly the same reasoning applies to protein crystallization (Rupp, 2010). It is known that the enthalpy change associated to the formation of protein crystals is not significant. On the other hand, proteins in crystals are structured and organized when compared to conformational freedom of proteins in solution, implying a loss of entropy when molecules from a given protein self-assemble into crystals. However, this reduction in entropy is over-compensated by the release of c. 5-30 water molecules which are freed from the surface of each protein into the highly entropic, surrounding aqueous medium in order to drive the process from protein in solution to protein is crystalline state (Rupp, 2010), i.e., $\Delta S_{\text {solvent }}>0, \Delta S_{\text {protein }}<0$, and $\left|\Delta S_{\text {solvent }}\right|>$ $\left|\Delta S_{\text {protein }}\right|$.

This scenario is analogous to black hole formation, because black holes are open systems too. Thus, clumping matter increases order during gravitational collapse and is responsible for the entropy "exported" into the surrounding space. Therefore, the entropy of the black hole environment needs to be included in the overall entropy computation (Adams and Laughlin, 1999).

At first sight, it may seem that life and black hole formation oppose or even violate the second law. Rather, they act together, because maintaining highly ordered structures allows for a global increase in entropy, something which would not take place in the absence of life and black holes. Therefore, neither life nor black hole formation violate the second law of thermodynamics (Lineweaver and Egan, 2008; Lineweaver, 2014).

Adapting these concepts to the "Evolving universe" hypothesis, once a growing $\mathrm{SMBH}$ (or a group of SMBHs occupying a given region of space) attains a critical density, a phase transition could take place: matter could adopt an ordered and structured form driven by an increase in entropy in the surrounding space. If blown into a newly born universe (either as a singularity or in a certain volume of space, thus reducing the dependence of the future universe on inflation), entropy in the parent universe could at least in part contribute to the future expansion and evolution of the offspring universe. One such event could eventually explain the cold spot (Figure 2A) as the leftover, surrounding space in the parent universe, although the recent findings reported by Mackenzie et al. (2017) suggest that a supervoid is unlikely to explain this mysterious low-temperature area in the CMB.

\section{Can an Isolated System Transit from a High Entropy to a Low Entropy Microstate?}

The statistical nature of the entropy concept introduced in 1872 by Ludwig Boltzmann allows transitions from high to low entropic states in isolated systems with no violation of the second law (Boltzman, 1872). To understand the basics 
behind this statement, the notion of fluctuation must be introduced. For a deeper understanding of this topic, the reader is recommended to review the $\mathrm{H}$-theorem, a natural consequence of the Boltzmann equation, the Boltzmann entropy curve and Poincaré recurrences, all of which lie beyond the scope of the present article.

Statistically, systems move from low to high entropy because there are many more unordered states than ordered states. However, since we are dealing with probabilities, sometimes systems move from high to low entropy, in a process known as a fluctuation. Obviously, it is incredibly more likely for something to move from low to high entropy than the opposite. Nevertheless, no matter how small it is, the probability of moving from higher to lower entropy is greater than zero, although it is expected for the fluctuation to take place over unimaginable timescales. Obviously, over very long time periods, the system will spend only a minute fraction of its time in one of these recurring states, Many of these low entropy fluctuations are exceedingly rare, and the lower the entropy goes, the rarer they are.

Let us go back to the glass of water containing ca. 6.023 $\times 10^{21}$ glucose molecules evenly distributed, but constantly moving in an unpredictable way. Entropy fluctuations will occur frequently. Thus, maximum entropy is achieved when half the glucose molecules are dissolved in each side of the imaginary membrane. However, it seems obvious that the erratic movement of the glucose molecules will easily cause minimum possible fluctuations corresponding to $6.023 \times 10^{21}$ +1 glucose molecules on one side of the glass membrane and to $6.023 \times 10^{21}-1$ glucose molecules on the othermeaning a tiny reduction in entropy. Occasionally, a few more glucose molecules will be found instantaneously on one side of the glass membrane, corresponding to larger reductions in entropy. The longer we wait, the larger the entropy fluctuation we will probably see. Eventually, if we wait long enough, we will see all the glucose molecules on one side of the glass membrane. Of course, the probable time required to observe this effect is truly enormous, many multiples of the age of the universe and, in practical terms, we might as well ignore this occurrence. Nevertheless, if/when it happens, the glucose molecules will quickly recover their equilibrium value of entropy, but given enough time, this same situation will happen again. In summary, given the statistical nature of the movement of glucose molecules in a high entropic state, transitions from high to low entropic states do occur with no violation of the second law. In other words, the second law of thermodynamics does not prevent the rare events which revert the direction of the entropy change.

\section{How Could the Universe Have Been Initiated with a Low Level of Entropy?}

It seems generally accepted that our universe was born with the Big Bang in a state of low entropy and that the entropy has increased ever since. Had the universe been born in a high entropy, equilibrium state, there would be no stars, planets or life (Lineweaver, 2014). But how was the universe initiated with a low level of entropy? A possible answer involves the concept of fluctuation and derives from the statistical nature of the entropy concept.

Assuming the universe was at thermal equilibrium at a given point in time, its entropy could not have increased further, so it would stay steady, except for fluctuations. As may be expected, larger fluctuations are exponentially less likely than smaller ones, but given enough time, every type of fluctuation will eventually happen.

Let us assume we start with a maximum entropy, "dead" configuration of the universe, where energy is no longer available to do any kind of work. Over tremendous timescales one expects the universe to move spontaneously into stages of considerably lower entropy, as clearly illustrated by Boltzmann in his entropy curve (Boltzmann, 1895, 1897). Although exceedingly rare, naturally occurring fluctuations may randomly occur which will lower the universe entropy enough to initiate a new cycle of inflation, conventional cosmology and "heat-death" (Dyson et al., 2002). The overwhelming majority of fluctuations will not reduce sufficiently entropy to a level capable of igniting a new cycle of inflation. Among the remaining fluctuations, many will probably lead to unviable universes, whereas others may originate universes rather different from our own. In other words, maybe our universe is currently in a state of fluctuation away from its normal equilibrium, meaning that the low entropy of the early universe may have been caused by a statistical accident.

\section{A LIVE UNIVERSE: ONE STEP BEYOND THE EVOLVING UNIVERSE}

\section{A Biological-Like Evolution of the Universe}

Quoting Hao Wei from the Beijing Institute of Technology, “The fate of our universe is an unceasing topic of philosophy and religion." We believe biology should also be considered in this discussion.

Going back to Smolin's "Evolving universe," a multiuniverse (or a family of multiple universes) may be envisaged as a hyperdimensional network where each parent universe budsoff offspring universes (Figure 3A). Similar families can be found in the natural, organic world, baker's yeast (Saccharomyces cerevisiae) for example, a fungus that buds-off for reproductive purposes (Figure 3B). Reproductive rates of Saccharomyces cerevisiae are obviously far apart from the cosmic timescale of black holes. But the budding mechanism, either micro- or macromanaged, looks the same. If the physical constants of the newborn universe (or the gene complement in next generation Saccharomyces cells) turn out well the infant universe can survive, thrive and eventually produce its own offspring. Otherwise the newly formed universe will be unviable, abort and eventually "die," in an only-the-fittest-survive scenario. Thus, after birth, i.e., Big Bang, each new universe if "healthy" (i.e., fit enough from a physics point of view) can undergo a juvenile phase of development before attaining maturity at the "budding off" reproductive phase.

Determining whether organisms retain some control on their own evolution remains a controversial issue in Biology. The way organisms assemble characters provides a good example. 


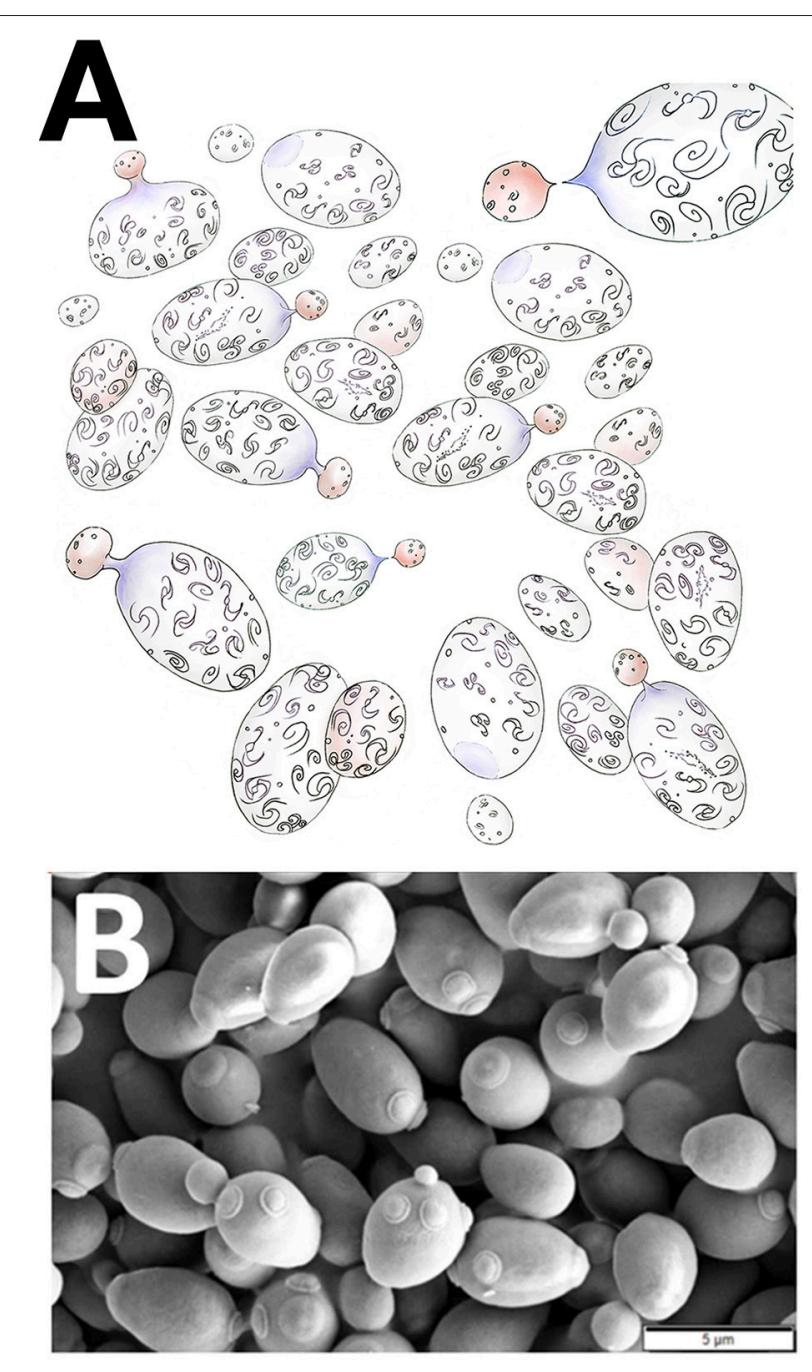

FIGURE 3 | Budding-off reproduction. (A) Artistic representation of a family of multiple universes where each parent universe buds-off offspring universes. Billions of galaxies are typically found in each universe. Note the scars left in "parent universes" by the previous budding-off of newly born universes (Art: Sofia Matalonga Jorge). (B) A colony of yeasts (Saccharomyces cerevisiae) actively budding-off for reproductive purposes. Note the scars left in mother yeast cells by the previous budding-off of newly born S. cerevisiae cells. Courtesy of Mogana Das Murtey and Patchamuthu Ramasamy (CC BY-SA 3.0, http://creativecommons.org/licenses/by-sa/3.0, via Wikimedia Commons. URL: https://commons.wikimedia.org/wiki/File \%3ASaccharomyces_cerevisiae_SEM.jpg).

In Biology, a character is any observable feature, or trait, of an organism. Characters may be acquired, if expressed in response to the environment, or inherited, if exhibited as a result of the expression of one or more genes transmitted from parents to offspring. Thus, both genome and environment directly shape the organism's phenotype (Alberts et al., 2002).

For a long time it seemed firmly established in Biology that acquired characters are not inherited. However, current evidence suggests that the environment may somehow influence the path of evolution by predisposing specific genes to mutation. Mutations are still somewhat random, but occur preferentially in certain genes which are, in turn, "selected" by the environment (Dias and Ressler, 2014). Indeed, organisms' genomes contain epigenetic marks that switch genes on and off. Changing these marks seems to alter the corresponding mutation rate for those genes. Thus, changes in epigenetic marks direct the preferential occurrence of mutations, meaning that an organism "can switch" genes on or off in order to adapt (Rönn et al., 2013). This process does not change the organism's overall genetic make-up by itself, but shapes the way natural selection acts upon those genes, directing evolution into more appropriate directions (Jaenisch and Bird, 2003; Aguilera et al., 2010; Kumar et al., 2013).

Beyond the fine-tuning of physical constants described in the previous section, an additional, finer level of tuning may be induced to be at play within the environment black holes "live in." The relative proportion of heavy elements or other environmental conditions affecting the functioning and structure of black holes might somehow influence or restrict the range of values the physical constants may "randomly" assume in the offspring universe, much in the same way as the environment in which each organic carbon-organism lives may signal/condition the genes which are most likely to undergo random mutations.

The biological-like evolution of a multiuniverse population would then occur if physical constants underwent (random or environmentally-conditioned) changes (or "mutations") when passing down from parent universe to offspring universe, and if the unstructured particles entering the newborn universe were allowed to carry some or all of those new features. This would mean of course a partial cosmic forgetfulness and, ultimately, physical constants on the brink of cosmic DNA.

\section{A Live Universe}

Defining life as we know it is by no means an easy task. There seem to be two basic properties determining if an object should be considered alive: metabolism (including the object's basic functions, biomass increase and reproduction) and motion (McKay, 2004). In addition, all living organisms are self-organizing open systems which require energy to maintain entropy low. Quoting Erwin Schrödinger (1944) on life, "It feeds on negative entropy." The capacity to evolve and become increasingly adapted to the surrounding environment seems to be an equally important feature inherent to life forms.

Citing Aron and Grossman (2013), ESA Planck detectors revealed birth, life and death of the cosmos. In fact, our universe seems to fulfill the premises underlying the concept of life in a way that could justify the notion of a "living universe": after birth (i.e., the Big Bang), the universe grows and develops, star ignition releases energy (as much as mitochondria do in yeast cells, by synthesizing ATP and other energy-rich nucleotides suitable to provide the energy required by numerous biological processes), the universe matures and ages (including formation of heavy elements and eventually the evolution of "organic" life itself), forms $\mathrm{SMBHs}$ and (the Evolving universe now coming into play) produces its own offspring before dying away as a dark, cold fade out.

The fine tuning of physical constants raises some questions in what concerns the putative natural evolution of the galactic yeast colony. The model described above for the evolving universe 
could be compared to biological asexual reproduction. We may go one step further and imagine the exchange of a set of physical constants between universes, generating occasional gamma-ray bursts (Retter and Heller, 2012), which raises a query: are physical constants exactly the same in all parts of our universe?

Although unstructured, the transferred material between universes should carry some properties from the parent universes, supporting, once again, a partial Bojowald's cosmic forgetfulness (Bojowald, 2007), in the way of chromosomal crossover. This exchange of matter and energy between different universes would mimic, to some extent, biological gene transfer. "Naturally," many of the resulting mixtures would originate nonviable solutions, poorly fit universes, or would not affect universe viability at all, yet some would definitely lead to increased universe fitness. Moreover, in the history of natural evolution, the diversity of living organisms was vigorously boosted by the advent of sexual reproduction. Could such a step in the timeline of universal evolution have taken/be taking/ever take place?

Organic life may also help reveal other similarities between the reproduction of living beings and reproduction of "living universes." Richard Dawkins's "non-discrete organisms" are suitable counterparts for such analogy. Non-discrete organisms are multicellular, differentiated beings whose offspring start out as single cells. Each newborn organism must then go through all stages of development before reaching maturity and reproductive differentiation, after which a new single cell is produced, ending one life cycle and starting a new one. Discrete organisms, by contrast, don't make use of single cells to pass from one generation to another, and reproduce by multiplying modules of their own bodies. According to Dawkins (1999), the following arguments support a greater complexity of nondiscrete organisms, in contrast to the simpler discrete organisms.

First, non-discrete organisms are able to grow a more complex physiology. While a discrete organism is maturing, improvements may positively interfere, creating a selective advantage over its peers. However, as a discrete organism lacks germinal cells, if the new improvement is not present in the next generation individuals, those species' individuals are bound to start from scratch in the next cycle, with no memory of previous improvements. Non-discrete organisms, on the other hand, retain memory of new improvements when the information is stored in germinal cells, allowing new information to be passed on to the next generation. Thus, over time additional improvements gradually accumulate, making tissues and organs in non-discrete organisms ever more complex. To simplify, complex organs such as the heart or the brain just cannot be made by a single genetic stroke of luck.

Secondly, setting up a developmental program allows for a rhythmic calendar to operate, which explains specific, essential steps observed in many developing embryos, such as in animals. Having a rhythmic calendar allows for more control mechanisms to take action, thus reducing the probability of error. Less error means more accuracy when growing into complexity.

Dawkins coined the passage of generations in non-discrete organisms through a single germinal cell a "bottleneck." The "bottleneck" model seems to fit into the "living universe" life cycle. Growth, maturation and aging with specific times and steps "bottleneck through" a singularity known as Big Bang, and a new "living universe" life cycle ensues. It also fits into Bojowald's cosmic forgetfulness, although an incomplete forgetfulness must be admitted. "Remembering" some of the parent universe's programming may be crucial for baby universes' physical constants' viability, while not remembering gives room to mutation and experimentation. Being non-discrete helps reiterating the experiment. So the universe lives.

To all intents and purposes, we may well be living in a "living universe," forming along other budding universes a multiuniverse family.

\section{CONCLUDING REMARKS}

The proposal of a "Living universe" embraces both the "Oscillating universe" and "Evolving universe" hypotheses. A $\mathrm{SMBH}$ or a region of very high density of matter in our universe (and thus of relatively lower entropy) could, under an immense gravitational field, give birth to a new universe (or inject matter into another pre-existent universe) in a phase transition involving cosmic forgetfulness, and leaving behind an area of emptier space (e.g., a cold spot; and thus of relatively higher entropy) in the parent universe. The whole process may be driven by an overall raise in entropy. Under these circumstances, the loss of matter in the parent universe may further contribute to an accelerated rate of expansion.

One may argue that hypothesizing on the universe being alive could be the product of forcing or adapting existing physical phenomena to fit the usual criteria for biological life. Nevertheless, it is still somewhat intriguing to find a parallelism between several astrophysical phenomena and biological life. Maybe new definitions for life and biological life should be pondered, since these analogies do exist. In this case, we must keep our minds wide open to other forms of life which may exist or thrive out there, be it in this or in any other universe.

Everything we see may be an infinitesimal part of a multiuniverse, naturally evolving to produce increasingly fit offspring universes toward a far reaching, unfathomable goal. Somewhere along this process, the evolution of intelligent beings may be (or maybe not!) an essential intermediate. However, if we drive ourselves to extinction, then human intelligence will be neither a leading trait nor even an intermediate contributing step to the future evolution of the universe. Or maybe other human-like attributes, with or without intelligence, will assume a prominent role.

Of course, we should not think ourselves (or any intelligent life form) at the top of evolution. Other levels/forms of intelligence or self-consciousness may lie ahead, impossible to predict by our "rudimentary" minds. We do have a long history of considering ourselves and/or our attributes at the center of everything. Alternatively, the continued evolution of "the galactic yeasts" may proceed through pathways which include neither ourselves nor our intelligence, in much the same way as dinosaurs were wiped out from the Earth, allowing the subsequent outbreak of mammals.

This manuscript was written by biologists with a strong interest in cosmology with the tentative goal of providing Cosmology with novel, biologically-related ideas, as well as incrementing the interest of the biological community in the 
astronomical sciences. In addition, the hypothesized life-style evolution of our universe most probably raises more questions than it settles. Its main purposes are therefore to stimulate discussion on some of the most debated issues of modern cosmology.

\section{REFERENCES}

Adams, F. C., and Laughlin, G. (1999). The Five Ages of the Universe: Inside the Physics of Eternity. New York, NY: The Free Press.

Aguilera, O., Fernández, A. F., Mu-oz, A., and Fraga, M. F. (2010). Epigenetics and environment: a complex relationship. J. Appl. Physiol. 109, 243-251. doi: 10.1152/japplphysiol.00068.2010

Alberts, B., Johnson, A., Lewis, J., Raff, M., Roberts, K., and Walter, P. (2002). Molecular Biology of the Cell, 4th Edn. New York, NY: Garland Science.

Aron, J., and Grossman, L. (2013). Planck map reveals birth, life and death of a cosmos. New Sci. 217, 8-9. doi: 10.1016/S0262-4079(13)60780-0

Azreg-Aïnou, M. (2015). Confined-exotic-matter wormholes with no gluing effects. Imaging supermassive wormholes and black holes. J. Cosmol. Astroparticle Phys. 07:037. doi: 10.1088/1475-7516/2015/07/037

Barrow, J. D. (2008). New Theories of Everything: The Quest for Ultimate Explanation. New York, NY: Oxford University Press.

Battersby, S. (2006), September 4). Top 10: Weirdest cosmology theories. New Scientist. Availabe online at: https://www.newscientist.com/article/dn9987top-10-weirdest-cosmology-theories/.

Begelman, M. C. (2003). Evidence for black holes. Science 300, 1898-1003. doi: $10.1126 /$ science. 1085334

Bojowald, M. (2007). What happened before the Big Bang? Nat. Phys. 3, 523-525. doi: 10.1038/nphys654

Boltzman, L. (1872). Weitere Studien über das Wärmegleichgewicht unter Gasmolekülen, Sitzungsber. Kais. Akad. Wiss. Wien Math. Naturwiss. 66, 275-370.

Boltzmann, L. (1895). On certain questions of the theory of gases. Nature 51, 413-415. doi: 10.1038/051413b0

Boltzmann, L. (1897). "On Zermelo's paper On the mechanical explanation of irreversible processes," in History of Modern Physical Sciences: Vol. 1, The Kinetic Theory of Gases. An Anthology of Classic Papers with Historical Commentary, ed N. S. Hall (London: Imperial College Press), $412-420$.

Buchdahl, H. A. (1970). Non-linear lagrangians and cosmological theory. Mon. Not. R. Astron. Soc. 150, 1-8. doi: 10.1093/mnras/150.1.1

Cai, Y.-C., Neyrinck, M. C., Szapudi, I., Cole, S., and Frenk, C. S. (2014). A possible cold imprint of voids on the microwave background radiation. Astrophys. J. 786:110. doi: 10.1088/0004-637X/786/2/110

Caldwell, R. R., Kamionkowski, M., and Weinberg, N. N. (2003). Phantom energy: dark energy with $\omega<-1$ causes a cosmic doomsday. Phys. Rev. Lett. 91:071301. doi: 10.1103/PhysRevLett.91.071301

Carr, B., and Elli, G. (2008). Universe or multiverse? Astron. Geophys. 49, 2.29-2.33. doi: 10.1111/j.1468-4004.2008.49229.x

Chensky, E. V. (2013). The oscillating universe theory (To the Unified Field Theory). Int. J. Astron. Astrophys. 3, 438-463. doi: 10.4236/ijaa.2013.34052

Cruz, M., Turok, N., Vielva, P., Martínez-González, E., and Hobson, M. (2007). A cosmic microwave background feature consistent with a cosmic texture. Science 318, 1612-1614. doi: 10.1126/science.1148694

Davies, P. C. W. (1982). The Accidental Universe. New York, NY: Cambridge University Press.

Davis, T. M. (2014). Cosmological constraints on dark energy. Gen. Relat. Gravit. 46:1731. doi: 10.1007/s10714-014-1731-1

Dawkins, R. (1999). The Extended Phenotype: The Long Reach of the Gene. Oxford: Oxford University Press.

Dias, B. G., and Ressler, K. J. (2014). Parental olfactory experience influences behavior and neural structure in subsequent generations. Nat. Neurosci. 17, 89-96. doi: 10.1038/nn.3594

\section{AUTHOR CONTRIBUTIONS}

Both RF and JF have made substantial, direct and intellectual contributions to the work, and approved it for publication.

Disconzi, M. M., Kephart, T. W., and Scherrer, R. J. (2015). New approach to cosmological bulk viscosity. Phys. Rev. D. 91:043532. doi: 10.1103/PhysRevD.91.043532

Dyson, L., Kleban, M., and Susskind, L. (2002). Disturbing implications of a cosmological constant. J. High. Energy Phys. 10:011. doi: 10.1088/1126-6708/2002/10/011

Easson, D. A., and Brandenberger, R. H. (2001). Universe generation from black hole interiors. J. High Energy Phys. JHEP06:24. doi: 10.1088/1126-6708/2001/06/024

Egan, C. A., and Lineweaver, C. H. (2010). A larger estimate of the entropy of the universe. Astrophys. J. 710, 1825-1834. doi: 10.1088/0004-637X/710/2/1825

Feeney, S. M., Johnson, M. C., Mortlock, D. J., and Peiris, H. V. (2011). First observational tests of eternal inflation. Phys. Rev. Lett. 107:071301. doi: 10.1103/PhysRevLett.107.071301

Fewster, C. J., and Roman, T. A. (2005). Problems with wormholes which involve arbitrarily small amounts of exotic matter. arXiv:gr-qc/0510079.

Friedmann, A. (1922). Über die krümmung des raumes. Z. Phys. 10, 377-386. doi: $10.1007 / \mathrm{BF} 01332580$

Gale, G. (1990). "Cosmological fecundity: theories of multiple universes," in Physical Cosmology and Philosophy (Philosophical Topics), ed J. Leslie (New York, NY: Prentice Hall), 189-206.

Haggard, H. M., and Rovelli, C. (2015). Quantum-gravity effects outside the horizon spark black to white hole tunneling. Phys. Rev. D 92:104020. doi: 10.1103/PhysRevD.92.104020

Hawking, S. W. (1975). Particle creation by black holes. Commun. Math. Phys. 43, 199-222. doi: 10.1007/BF02345020

Hawking, S. W., Perry, M. J., and Strominger, A. (2016). Soft hair on black holes. Phys. Rev. Lett. 116:231301. doi: 10.1103/PhysRevLett.116.231301

Hayward, S. A. (1999). Dynamic wormholes. Int. J. Mod. Phys. D 8, 373-382. doi: $10.1142 /$ S0218271899000286

Hubble, E. P. (1929). A relation between distance and radial velocity among extra-galactic nebulae. Proc. Natl. Acad. Sci. U.S.A. 15, 168-173. doi: 10.1073/pnas.15.3.168

Inoue, K. T., and Silk, J. (2006). Local voids as the origin of largeangle cosmic microwave background anomalies. Astrophys. J. 648, 23-30. doi: $10.1086 / 505636$

Jaenisch, R., and Bird, A. (2003). Epigenetic regulation of gene expression: how the genome integrates intrinsic and environmental signals. Nat. Genet. 33, 245-254. doi: $10.1038 / \mathrm{ng} 1089$

Jaffe, T. R., Banday, A. J., Eriksen, H. K., Górski, K. M., and Hansen, F. K. (2005). Evidence of vorticity and shear at large angular scales in the WMAP data: a violation of cosmological isotropy? Astrophys. J. 629, L1-L4. doi: 10.1086/444454

Khoury, J., Ovrut, B. A., Steinhardt, P. J., and Turok, N. (2001). The Ekpyrotic universe: colliding branes and the origin of the hot big bang. Phys. Rev. D 64:123522. doi: 10.1103/PhysRevD.64.123522

Kim, S.-W., and Park, M.-I. (2015). Black hole as a wormhole factory. Phys. Lett. B 751, 220-226. doi: 10.1016/j.physletb.2015.10.045

Kragh, H. (2009). Contemporary history of cosmology and the controversy over the multiverse. Ann. Sci. 66, 529-551. doi: 10.1080/00033790903047725

Kragh, H. (2013). Cyclic models of the relativistic universe: the early history. arXiv:1308.0932v1.

Kumar, S., Kumari, R., and Sharma, V. (2013). Roles, and establishment, maintenance and erasing of the epigenetic cytosine methylation marks in plants. J. Genet. 92, 629-666. doi: 10.1007/s12041-013-0273-8

Levinthal, C. (1968). Are there pathways for protein folding? J. Chim. Phys. 65, 44-45. doi: 10.1051/jcp/1968650044 
Lineweaver, C. H. (2014). "The entropy of the universe and the maximum entropy production principle," in Beyond the Second Law, Understanding Complex Systems, eds R. C. Dewar, C. H. Lineweaver, R. K. Niven, and K. Regenauer-Lieb (Berlin: Springer-Verlag), Chapter 22, 415-427.

Lineweaver, C. H., and Egan, C. (2008). Life, gravity and the second law of thermodynamics. Phys. Life Rev. 5, 225-242. doi: 10.1016/j.plrev.2008.08.002

Lobo, F. S. N., and Oliveira, M. A. (2009). Wormhole geometries in $\mathrm{f}(\mathrm{R})$ modified theories of gravity. Phys. Rev. D 80:104012. doi: 10.1103/PhysRevD.80.104012

Mackenzie, R., Shanks, T., Bremer, M. N., Cai, Y.-C., Gunawardhana, M. L. P., Kovács, A., et al. (2017). Evidence against a supervoid causing the CMB Cold Spot. Mon. Not. R. Astron. Soc. arXiv:1704.03814v1.

Marcos-Caballero, A., Fernández-Cobos, R., Martínez-González, E., and Vielva, P. (2016). On the void explanation of the Cold Spot. Mon. Not. R. Astron. Soc. 460, L15-L19. doi: 10.1093/mnrasl/slw063

McEwen, J. D., Feeney, S. M., Johnson, M. C., and Peiris, H. V. (2012). Optimal filters for detecting cosmic bubble collisions. Phys. Lett. D 2012:85103502. doi: 10.1103/PhysRevD.85.103502

McKay, C. P. (2004). What is life-and how do we search for it in other worlds? PLoS Biol. 2:e302. doi: 10.1371/journal.pbio.0020302

Morris, M. S., and Thorne, K. S. (1988). Wormholes in spacetime and their use for interstellar travel: a tool for teaching general relativity. Am. J. Phys. 56, 395-412. doi: $10.1119 / 1.15620$

Nadathur, S., and Crittenden, R. (2016). A detection of the integrated Sachs-Wolfe imprint of cosmic superstructures using a matched-filter approach. Astrophys. J. Lett. 830:L19. doi: 10.3847/2041-8205/830/1/L19

Nadathur, S., Lavinto, M., Hotchkiss, S., and Räsänen, S. (2014). Can a supervoid explain the Cold Spot? Phys. Rev. D 90:103510. doi: 10.1103/PhysRevD.90.103510

Novikov, I. D. (1964). Delayed explosion of a part of the Fridman universe and quasars. Astron. Zh. 41, 1075-1083.

Perlmutter, S., Aldering, G., Goldhaber, G., Knop, R. A., Nugent, P., Castro, P. G., et al. (1999). Measurements of omega and lambda from 42 high-redshift supernovae. Astrophys. J. 517, 565-586. doi: 10.1086/307221

Poplawski, N. J. (2010). Radial motion into an Einstein-Rosen bridge. Phys. Lett. B 687, 110-113. doi: 10.1016/j.physletb.2010.03.029

Pourhasan, R., Afshordi, N., and Mann, R. B. (2014). Out of the white hole: a holographic origin for the big bang. J. Cosmol. Astropart. Phys. 1404:005. doi: 10.1088/1475-7516/2014/04/005

Press, W. H., Lightman, A. P., Peierls, R., and Gold, T. (1983). Dependence of macrophysical phenomena on the values of the fundamental constants. Philos. Trans. R. Soc. Lond. A 310, 323-336. doi: 10.1098/rsta.1983.0094

Regge, T., and Wheeler, J. A. (1957). Stability of a Schwarzschild singularity. Phys. Rev. 108, 1063-1069. doi: 10.1103/PhysRev.108.1063

Retter, A., and Heller, S. (2012). The revival of white holes as small bangs. New Astron. 17, 73-75. doi: 10.1016/j.newast.2011.07.003

Riess, A. G., Filippenko, A. V., Challis, P., Clocchiatti, A., Diercks, A., Garnavich, P. M., et al. (1999). Observational evidence from supernovae for an accelerating universe and a cosmological constant. Astron. J. N.Y. 116, 1009-1038. doi: 10.1086/300499

Riess, A. G., Macri, L. M., Hoffmann, S. L., Scolnic, D., Casertano, S., Filippenko, A. V., et al. (2016). A 2.4\% determination of the local value of the Hubble constant. Astrophys. J. 826:56. doi: 10.3847/0004-637X/826/1/56
Rönn, T., Volkov, P., Davegårdh, C., Dayeh, T., Hall, E., Olsson, A. H., et al. (2013). A six months exercise intervention influences the genome-wide DNA methylation pattern in human adipose tissue. PLoS Genet. 9:e1003572. doi: 10.1371/journal.pgen.1003572

Rupp, B. (2010). Biomolecular Crystallography: Principles, Practice, and Application to Structural Biology. New York, NY: Garland Science.

Sánchez, J. C. B. (2014). The inflationary origin of the Cold Spot anomaly. Phys. Lett. B 739, 269-278. doi: 10.1016/j.physletb.2014.11.001

Schrödinger, E. (1944). What Is Life? The Physical Aspect of the Living Cell. Cambridge: Cambridge University Press.

Scrimgeour, M. I., Davis, T., Blake, C., James, J. B., Poole, G. B., StaveleySmith, L., et al. (2012). The WiggleZ dark energy survey: the transition to large-scale cosmic homogeneity. Mon. Not. R. Astron. Soc. 425, 116-134. doi: 10.1111/j.1365-2966.2012.21402.x

Shinkai, H.-A., and Hayward, S. A. (2002). Fate of the first traversible wormhole: black-hole collapse or inflationary expansion. Phys. Rev. D D66:044005. doi: 10.1103/PhysRevD.66.044005

Smolin, S. (1997). The Life of the Cosmos. Oxford: Oxford University Press.

Szapudi, I., Kovács, A., Granett, B. R., Frei, Z., Silk, J., Burgett, W., et al. (2015). Detection of a supervoid aligned with the cold spot of the cosmic microwave background. Mon. Not. R. Astron. Soc. 450, 288-294. doi: 10.1093/mnras/ stv488

Thomas, J., Ma, C.-P., McConnell, N. J., Greene, J. E., Blakeslee, J. P., and Janish, R. (2016). A 17-billion-solar-mass black hole in a group galaxy with a diffuse core. Nature 532, 340-342. doi: 10.1038/nature17197

Tolman, R. C. (1934). Relativity, Thermodynamics and Cosmology. Oxford: Clarendon Press.

Venemans, B. (2015). Cosmology: a giant in the young universe. Nature 518, 490-491. doi: 10.1038/518490b

Visser, M. (1995). Lorentzian Wormholes: From Einstein to Hawking. New York, NY: AIP Press.

Weinberg, S. (1977). The First Three Minutes: A Modern View of the Origin of the Universe. London: Trinity Press.

Wu, W. L. K., Ade, P. A. R., Ahmed, Z., Alexander, K. D., Amiri, M., Barkats, D., et al. (2016). Initial performance of BICEP3: a degree angular scale $95 \mathrm{GHz}$ band polarimeter. J. Low Temp. Phys. 184, 765-771. doi: 10.1007/s10909-015-1403-x

Wu, X.-B., Wang, F., Fan, X., Yi, W., Zuo, W., Bian, F., et al. (2015). An ultraluminous quasar with a twelve-billion-solar-mass black hole at redshift 6.30. Nature 518, 512-515. doi: 10.1038/nature14241

Zwanzig, R., Szabo, A., and Bagchi, B. (1992). Levinthal's paradox. Proc. Natl. Acad. Sci. U.S.A. 89, 20-22. doi: 10.1073/pnas.89.1.20

Conflict of Interest Statement: The authors declare that the research was conducted in the absence of any commercial or financial relationships that could be construed as a potential conflict of interest.

Copyright (c) 2017 Ferreira and Ferreira. This is an open-access article distributed under the terms of the Creative Commons Attribution License (CC BY). The use, distribution or reproduction in other forums is permitted, provided the original author(s) or licensor are credited and that the original publication in this journal is cited, in accordance with accepted academic practice. No use, distribution or reproduction is permitted which does not comply with these terms. 